\title{
Estimated Prevalence of Immunity to Poliomyelitis in the City of São Paulo, Brazil: a Population-Based Survey
}

\author{
Carlos Roberto Veiga Kiffer, Orlando Jorge Conceição, \\ Edgar Bortholi Santos, Ester Sabino and Roberto Focaccia
}

\author{
Institute of Infectology Emilio Ribas, \\ University of São Paulo, São Paulo, SP, Brazil
}

\begin{abstract}
Objectives. Estimate the prevalence of immunity to poliomyelitis (anti-polio antibodies) in the city of São Paulo/Brazil through a population-based survey. Methods. A quantitative and inductive method was used to draw a representative sample of the population. Randomization and stratification (based on sex, age and residence region) was done, and 1,059 individuals were studied on a homevisit basis (structured questionnaires and blood samples). A microneutralization test was performed to detect anti-polio antibodies against serotypes 1,2 and 3. Results. The estimated prevalence of immunity to poliomyelitis was high, with $94.6 \%$ prevalence of anti-polio 1 antibodies, $\mathbf{9 8 . 8 \%}$ anti-polio 2 and $91.9 \%$ anti-polio 3 . Despite this high prevalence, there were significantly lower prevalence levels in some groups, specially among age and residence region groups. Discussion. Routine child immunization and NIDs with OPV have provided excellent levels of serological immunity to poliomyelitis in the population of the city of São Paulo, Brazil. However, there may be specific groups with a lower prevalence of immunity. Estimations of the prevalence of immunity to poliomyelitis were made in a population-based survey, which could be used as an auxiliary tool for supporting the polio eradication program.

Key Words: Immunity prevalence, poliomyelitis, poliviruses, seroepidemiologic studies, antibodies, viral blood.
\end{abstract}

The World Health Organization (WHO) decided in 1988 to adopt as a goal the eradication of poliomyelitis caused by wild Poliovirus by the year 2000 . In order to achieve this goal, three strategies were adopted: maintenance of a high degree of vaccination coverage, application of supplementary vaccine doses during national vaccination days and development of surveillance programs (epidemiological and laboratory)

Received on 10 June 2002; revised 25 October 2002.

Address for correspondence: Dr. Carlos Roberto Veiga Kiffer Alameda dos Guaicanãs 984, Planalto Paulista / São Paulo / Brazil / Zip Code - 04064-031. Phone: +55 1155853180 / Fax: +551150787041/E-mail: kiffer@uninet.com.br

Manuscript Presented in part at:

$1^{\text {st }}$ Portuguese-Galician Congress, $5^{\text {th }}$ National Congress, $1^{\text {st }}$ Academic Meeting SÃo Paulo-Santiago de Compostela-Porto of Infectious Diseases. $15^{\text {th }}$ to $19^{\text {th }}$ October, $2000\left(2^{\text {nd }}\right.$ prize award winner).

The Brazilian Journal of Infectious Diseases 2002;6(5):232-243 (C) 2002 by The Brazilian Journal of Infectious Diseases and Contexto Publishing. All rights reserved.

$1413-8670$
[1]. Monitoring vaccine coverage is used as a measure of program progress, while monitoring disease incidence through surveillance programs (either with acute flaccid paralysis - AFP - surveillance or viral isolation) is used as a measure of its impact [2]. These measures have been proven extremely efficient and there has been a considerable reduction in the number of cases reported worldwide during the last decade [37]. Even before the WHO decision, the Pan-American Health Organization (PAHO) presented an even more audacious proposal, with the goal of interrupting thetransmission of wild Poliovirus in the Americas by 1990 [8]. Through a combination of an increment in vaccination coverage and rigorous surveillance, a drastic reduction in the number of poliomyelitis cases in the American continent was achieved. In 1994, the International Commission for the Certification of Poliomyelitis Eradication concluded that the autochthonous transmission of wild Poliovirus had been eradicated in the Americas [9]. Furthermore, new achievements have been constantly announced. In 
2000, the Western Pacific region was also certified free from wild Poliovirus transmission by the WHO, and an apparent global interruption of wild Poliovirus type 2 transmission was recently reported [10, 11].

However, wild Poliovirus is still a serious public health problem in several countries [10, 12-14]. Unfortunately, it is not possible to consider any given region completely free of the risk of reintroduction of wild Poliovirus while other regions of the world still have any endemic level. Therefore, the risk of its reintroduction in environments considered free must still be taken into consideration, especially in places where vaccination programs are irregular or where data on vaccine coverage are deficient [10].

In view of those facts, it is fundamental that we strive to extend to other continents the success obtained with the eradication program in the Americas. The ultimate goals of the poliomyelitis eradication program are total interruption of wild Poliovirus transmission and zero poliomyelitis incidence. In order to achieve these goals, the most efficient manner to eradicate transmission of wild polioviruses is to maintain both high vaccine coverage and an adequate system of epidemiological (AFP) and laboratory (viral isolation) surveillance [2, $3,15,16]$.

\section{Objectives}

The primary objective of this study was to estimate the prevalence of immunity to poliomyelitis (anti-polio neutralizing antibodies against serotypes 1,2 and 3) in the city of São Paulo/Brazil, the second largest urban center of the Americas. In order to achieve this goal, a population-based survey was made by sampling a representative population. This kind of study is potentially extendible to the public health system as an auxiliary tool for supporting poliomyelitis eradication.

\section{Material and Methods}

This research was carried out in 1996/1997, with previous Ethics Committee approval granted by the Instituto de Infectologia Emílio Ribas - São Paulo/ Brazil. Participation was voluntary and each participant or legal guardian signed a consent form, after being properly informed.

Sampling and Population. A quantitative design, with an inductive method of predicting statistical inferences, was applied, making use of a representative sample of the population of São Paulo city, Brazil. This sampling scheme was structured, based on official data on population distribution, according to sex, age and region (city districts) [17-19]. The population sample was drawn at random and stratified according to sex, age and residence region. It was co-conducted by the Datafolha Institute, a private polling research and statistics institute, with a residence-based interview (structured questionnaire) and blood collection.

The city districts were grouped into five geographical regions: North, South, East, West and Downtown. Sampling was carried out in two stages:

1. In the first stage, blocks previously classified into three different population levels - high, medium and low household density - were randomly drawn, giving the same draw probability for each household, based on the household density in each block. The region formed by blocks adjacent to the ones randomly drawn was denominated a "cluster".

2. In the second stage, households were randomly drawn within each block. The number of households per cluster was similar, since the proportion in relation to the population size had been taken into consideration.

Blocks and clusters were covered in a counterclockwise and centrifugal direction. Home visits were always made at at every third residence, both in cases of refusal and of acceptance to participate. Whenever an individual in a given residence refused to take part in this study, he/she could only be replaced by another person with the same age and sex characteristics (from the third residence in the same block). In collective residences (i.e. apartment buildings, condominiums, slums and alleys) a maximum of two homes were included. Only one individual per residence participated. 
The study population was restricted to individuals more than two years old, who had been living for more than two years in the city of São Paulo. Questionnaires were applied and blood samples were collected during visits to the homes. A system of quotas per age group was used, in order to guarantee a representative sampling of each age. One thousand and fifty nine $(1,059)$ individuals were studied in the following age groups (in years $)$ : $2-4(\mathrm{n}=61), 5-9(\mathrm{n}=108), 10-14(\mathrm{n}=104), 15$ $17(\mathrm{n}=58), 18-29(\mathrm{n}=250), 30-39 \quad(\mathrm{n}=184), 40-49$ $(n=123), 50-59(n=81)$ and 60 or more $(n=90)$.

All questionnaires were allocated a code that also identified the respective blood sample, and the information obtained was kept in secret by the investigators. Five teams simultaneously conducted home visits from February 25 to May 5, 1996, during weekends and holidays, in order to provide a representative sample [20].

Specimen testing. Blood samples were collected in coded sterile tubes and kept in an ice-filled box until they were taken to the laboratory. Sera were separated and kept in $\mathrm{a}-70^{\circ} \mathrm{C}$ freezer until testing, which was done at a central reference laboratory. A microneutralization test was performed to detect antipolio antibodies against serotypes 1, 2 and 3 . The method that was used is a standard procedure for measuring immunity to Poliovirus, described in the Manual of Laboratory Methods of the WHO Global Program for Vaccines and Immunization [21].

Briefly, sera were inactivated and twofold dilution ranges were made of samples in a test medium. After making the dilutions of the serum samples, equal amounts of diluted serum and challenge poliovirus [approximately $100 \mathrm{TCID}_{50}-(50 \%$ tissue culture infective dose)] were mixed and incubated at $36^{\circ} \mathrm{C}$ in microtiter well plates. After the first incubation period, the cell substrate (Hep-2) was added to the wells containing the serum-virus mixture. Then these mixtures were incubated at $36^{\circ} \mathrm{C}$ and observed for 5 days in order to determine (with a microscope) if there were cytopathic effects. A working reference serum with known poliovirus neutralizing capacity for all serotypes was tested in parallel to confirm the validity of the test.
Titers of the positive sera did not vary more than twofold from their median titers and the negative serum did not show any degree of neutralization.

The serum antibody titer was the highest serum dilution that protected $50 \%$ of cultures against 100 $\mathrm{TCID}_{50}$ of challenge virus. An anti-polio (serotypes 1 , 2,3 ) antibody titer $=8$, expressed as a reciprocal dilution, was considered positive, indicating immunity to poliomyelitis.

Statistical analysis. The estimated prevalence $(p)$ was defined as the number of individuals immune to poliomyelitis for each poliovirus type (presence of antipolio neutralizing antibodies to serotypes 1,2 or 3) divided by the total sample size (n). Standard deviations (SD), standard errors (not indicated here, available on request) and $95 \%$ confidence interval (where $95 \% \mathrm{CI}=p+/-1.96 \sqrt{\frac{p(1-p)}{n}}$ were calculated, without considering losses. Error margins were limited to $+/-3 \%$ for every occasionally intervening variable. Losses were equal among groups and the population maintained its representativity.

The significances of the group comparisons within the same strata, were determined by univariate analysis, using a difference test between proportions (one way analysis of variance); $\mathrm{P}<0.05$ were defined as the cutoff for statistical significance for a given comparison. A multivariate analysis was subsequently performed with a logistic regression model (SAS, version 8.01, SAS Institute Inc., North Carolina, USA), including the three stratification variables (age, sex and residence region). This analysis was used to determine significance $(\mathrm{P}<0.05)$ and Odds Ratio $(\mathrm{OR})$ of each variable in the model, separately [22].

\section{Results}

Graph 1 shows the estimated prevalence of immunity to poliomyelitis in the general population of the city of São Paulo, Brazil. A high prevalence of anti-polio immunity was observed in this population, with $94.6 \%$ anti-polio $1,98.8 \%$ anti-polio 2 and $91.9 \%$ anti-polio 3 immunity. Graph 2 shows the estimated prevalence of immunity to poliomyelitis 
Figure 1. Estimated prevalence of poliomyelitis immunity in São Paulo - Brazil

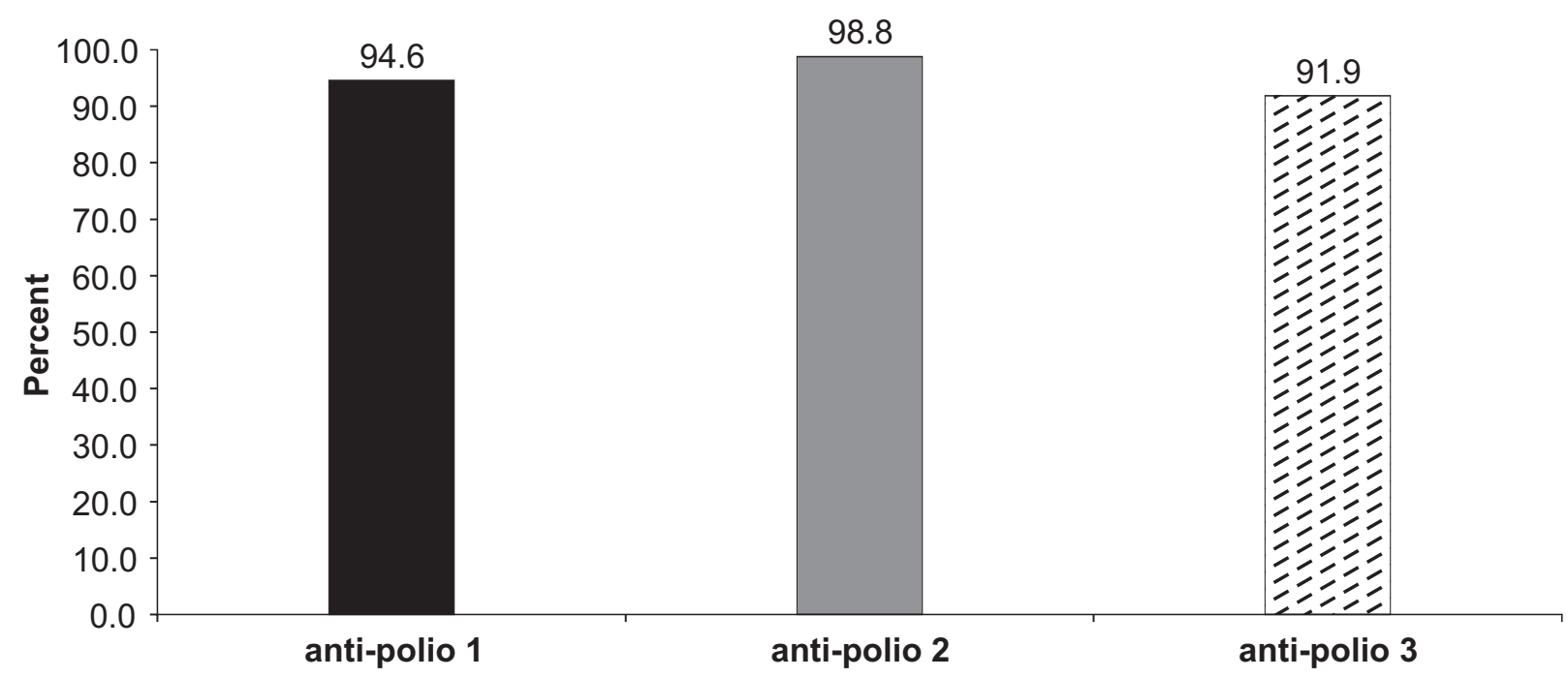

Figure 2. Estimated prevalence of poliomyelitis immunity per age group in São Paulo, Brazil

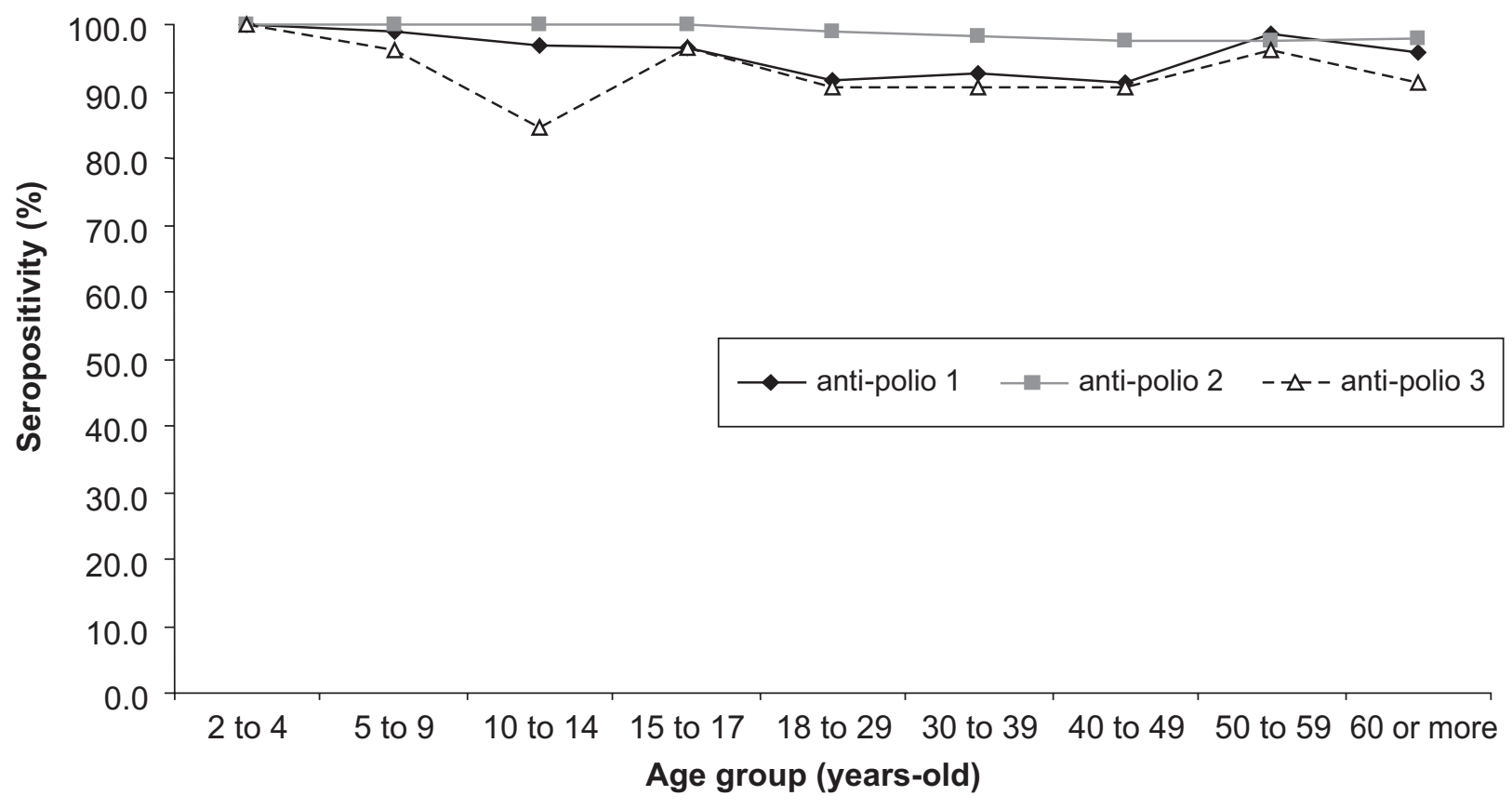


Table 1a. Estimated prevalence $(p)$ of anti-polio 1 immunity - Univariate analysis

\begin{tabular}{lrcccc}
\hline & $\mathbf{N}$ & $\boldsymbol{p}$ & SD & $\mathbf{9 5 \%} \mathbf{C I}$ & P value \\
\hline $\begin{array}{l}\text { Age } \\
\quad<18\end{array}$ & 343 & 0.977 & 0.151 & $0.961-0.993$ & 0.002 \\
$\quad{ }^{3} 18$ & 697 & 0.931 & 0.253 & $0.912-0.950$ & \\
Sex & & & & & \\
$\quad$ Men & 496 & 0.923 & 0.266 & $0.900-0.947$ & 0.002 \\
$\quad$ Women & 544 & 0.967 & 0.179 & $0.952-0.982$ & \\
Region & & & & & \\
$\quad$ North & 181 & 0.945 & 0.229 & $0.911-0.978$ & \\
$\quad$ East & 355 & 0.938 & 0.241 & $0.913-0.963$ & 0.898 \\
$\quad$ South & 293 & 0.949 & 0.220 & $0.923-0.974$ & \\
$\quad$ Downtown & 62 & 0.952 & 0.216 & $0.897-1.000$ & \\
$\quad$ West & 149 & 0.960 & 0.197 & $0.928-0.992$ & \\
\hline
\end{tabular}

Table 1b. Estimated prevalence of anti-polio 1 immunity - Multivariate analysis

\begin{tabular}{lcccc}
\hline & Estimate & SE & $\boldsymbol{\chi 2}$ & P value \\
\hline Age & 1.155 & 0.390 & 8.785 & 0.003 \\
Sex & -0.892 & 0.295 & 9.165 & 0.003 \\
& & & & \\
Region & - & - & 1.351 & 0.853 \\
North & 0.071 & 0.309 & 0.054 & 0.817 \\
East & 0.248 & 0.246 & 1.015 & 0.314 \\
South & 0.053 & 0.271 & 0.038 & 0.845 \\
Downtown & -0.105 & 0.494 & 0.045 & 0.833 \\
West & -0.267 & 0.369 & 0.525 & 0.469 \\
\hline
\end{tabular}

Table 1c. Estimated prevalence of anti-polio 1 immunity - Odds Ratio

\begin{tabular}{lcc}
\hline & OR & $\mathbf{9 5 \%}$ CI \\
\hline Age & 3.173 & $1.479-6.810$ \\
Sex & 2.439 & $1.370-4.348$ \\
Region & NA & NA \\
\hline
\end{tabular}

NA - not applicable. 
Table 2a. Estimated prevalence ( $p$ ) of anti-polio 2 immunity - Univariate analysis

\begin{tabular}{lrrlrr}
\hline & $\mathbf{N}$ & $\boldsymbol{p}$ & \multicolumn{1}{c}{ SD } & $\mathbf{9 5 \%} \mathbf{C I}$ & P value \\
\hline Age & & & & & \\
$\quad<18$ & 343 & 1.000 & 0.000 & $1.000-1.000$ & 0.011 \\
$\quad 18$ & 696 & 0.981 & 0.136 & $0.971-0.991$ & \\
$\quad$ Sex & & & & & \\
$\quad$ Men & 496 & 0.984 & 0.126 & $0.973-0.995$ & 0.317 \\
$\quad$ Women & 543 & 0.991 & $9.560 \mathrm{E}-02$ & $0.983-0.999$ & \\
Region & & & & & \\
$\quad$ North & 180 & 0.967 & 0.180 & $0.940-0.993$ & \\
$\quad$ East & 355 & 0.994 & $7.495 \mathrm{E}-02$ & $0.986-1.000$ & \\
$\quad$ South & 293 & 0.993 & $8.248 \mathrm{E}-02$ & $0.984-1.000$ & 0.029 \\
$\quad$ Downtown & 62 & 0.968 & 0.178 & $0.922-1.000$ & \\
$\quad$ West & 149 & 0.993 & $8.192 \mathrm{E}-02$ & $0.980-1.000$ & \\
\hline
\end{tabular}

Table 3a. Estimated prevalence ( $p$ ) of anti-polio 3 immunity - Univariate analysis

\begin{tabular}{lrcccc}
\hline & $\mathbf{n}$ & $\boldsymbol{p}$ & SD & $\mathbf{9 5 \%}$ CI & P value \\
\hline Age & & & & & \\
$\quad<18$ & 341 & 0.933 & 0.251 & $0.906-0.959$ & 0.266 \\
$\quad 18$ & 697 & 0.913 & 0.283 & $0.892-0.936$ & \\
Sex & & & & & \\
$\quad$ Men & 495 & 0.905 & 0.293 & $0.879-0.931$ & 0.114 \\
$\quad$ Women & 543 & 0.932 & 0.252 & $0.911-0.953$ & \\
Region & & & & & \\
$\quad$ North & 181 & 0.945 & 0.229 & $0.911-0.978$ & \\
East & 354 & 0.955 & 0.208 & $0.933-0.976$ & 0.001 \\
$\quad$ South & 292 & 0.878 & 0.329 & $0.839-0.915$ & \\
$\quad$ Downtown & 62 & 0.855 & 0.355 & $0.765-0.945$ & \\
$\quad$ West & 149 & 0.913 & 0.283 & $0.867-0.959$ & \\
\hline
\end{tabular}


Table 3b. Estimated prevalence of anti-polio 3 immunity - Multivariate analysis

\begin{tabular}{lcccc}
\hline & Estimate & SE & $\boldsymbol{\chi 2}$ & P value \\
\hline Age & 0.270 & 0.257 & 1.100 & 0.294 \\
Sex & -0.388 & 0.232 & 2.807 & 0.094 \\
Region & - & - & 17.202 & 0.002 \\
$\quad$ North & -0.451 & 0.284 & 2.527 & 0.112 \\
East & 0.651 & 0.237 & 7.551 & 0.006 \\
South & 0.447 & 0.190 & 5.554 & 0.018 \\
Downtown & 0.614 & 0.309 & 3.942 & 0.047 \\
West & 0.042 & 0.260 & 0.026 & 0.872 \\
\hline
\end{tabular}

Table 3c. Estimated prevalence of anti-polio 3 immunity - Odds Ratio

\begin{tabular}{lcc}
\hline & OR & $\mathbf{9 5 \%}$ CI \\
\hline Age & 1.310 & $0.791-2.169$ \\
Sex & 0.678 & $0.431-1.068$ \\
Region & & \\
$\quad$ East vs. South & 3.000 & $1.627-5.533$ \\
East vs. Downtown & 3.544 & $1.484-8.459$ \\
South vs. Downtown & 1.181 & $0.535-2.608$ \\
$\quad$ North vs. South & 2.456 & $1.185-5.090$ \\
$\quad$ North vs. Downtown & 2.900 & $1.116-7.539$ \\
\hline
\end{tabular}

for each age group; no age group had less than $84 \%$ estimated immunity prevalence.

Estimated prevalence of anti-polio 1 immunity. Univariate analysis showed a significantly lower prevalence of anti-polio 1 immunity among the male population $(\mathrm{P}=0.002)$. Among the age groups, $100 \%$ of the children up to 6 years old $(n=88)$ were immune. Furthermore, individuals under 18 years old were more frequently immune than those 18 years or older (univariate analysis, $\mathrm{P}=0.002$ )
(Table 1a). Additionally, there was a a significant difference among the age groups $<18,18-49$ and $\geq 50$ years-old $(\mathrm{P}<0.0001)$. A lower estimated prevalence of anti-polio 1 immunity was detected in the $18-49$ age group $(0.919,95 \% \mathrm{CI}=0.896-$ $0.942)$ comparedto the $<18(0.981,95 \% \mathrm{CI}=$ $0.966-0.996)$ and to the $\geq 50(0.971,95 \% \mathrm{CI}=$ 0.946-0.996) age groups.

Multivariate analysis indicated significance for both age and sex, independently $(\mathrm{P}=0.003$ for both), but not for residence region $(\mathrm{P}=0.716)$ (Table $1 \mathrm{~b})$. 
Furthermore, OR was 3.124 for the $<18$ year-old age group in comparison with the $\geq 18$ year-old group, $(95 \% \mathrm{CI}=1.457-6.697)$ and $\mathrm{OR}=2.439$ for females, in comparison with males $(95 \% \mathrm{CI}=$ 1.370-4.348) (Table 1c).

Estimated prevalence of immunity anti-polio 2. There was no significant difference in anti-polio 2 immunity between males and females (univariate analysis). However there were significant differences among residence regions $(\mathrm{P}=0.029)$ (Table 2a); the lowest prevalence was in the North region. Among age groups, children up to 6 years old were $100 \%$ immune $(n=88)$. Moreover, individuals under 18 years old were more frequently immune than those 18 years or older $(\mathrm{P}=0.011)$ (Table 2a).

Multivariate analysis was inadequate to evaluate any correlation for anti-polio 2 immunity, since this subgroup had the highest estimated immunity prevalence (98.8\%) (Figure 1).

\section{Estimated prevalence of anti-polio 3 immunity.} There was no significant difference between male and female anti-polio 3 immunity prevalances. However, there was a significant difference among residence regions $(\mathrm{P}=0.001)$; the lowest prevalence was found in the South and Downtown regions (Table 3a). Among the age groups, children up to 6 years old were $100 \%$ immune $(n=87)$.

Residence region had a significant effect on antipolio 3 immunity prevalence $(\mathrm{P}=0.002)$. The East, South and Downtown regions were significantly different $(\mathrm{P}=0.006,0.018$ and 0.047 , respectively). No significant differences were found among the age and sex groups (Table 3b). The OR values for the East region compared to South and Downtown were $3.000(95 \% \mathrm{CI}=1.627-5.533)$ and $3.544(95 \%$ $\mathrm{CI}=1.484-8.459)$, respectively (Table $3 \mathrm{c}$ ).

Although the population was not stratified according to color/race, all racial groups had a high immunity prevalence and no significant differences among these groups were observed with univariate analysis. No further analysis was performed.

\section{Discussion}

Despite the important achievements worldwide in the polio eradication initiative, particularly the recent eradication of wild Poliovirus in the Western Pacific region and the apparent global interruption of wild Poliovirus type 2 transmission [10, 11], wild Poliovirus transmission is still a severe public health threat in various regions of the world, particularly in Africa and South-east Asia [10, 12-14]. The continued circulation of wild Poliovirus types 1 and 3 poses a reintroduction risk for any polio-free region [10]. Thus, it is fundamental that all countries keep up with polio eradication and control strategies, even in regions considered free of wild poliovirus transmission. Furthermore, various areas with low OPV coverage have documented the circulation of OPV-derived and recombinant Poliovirus, including alarming recent outbreaks in the Dominican Republic and Haiti [16, 2325]. It is known that the key factor for controlling circulating OPV-derived viruses as well as wild polioviruses is achieving and maintaining high vaccination coverage, as recently reemphasized during the certification of poliomyelitis eradication in the Western Pacific Region [10, 16]. As a result, it is of decisive importance to follow adequate control measures through high vaccination coverage and adequate surveillance in order to achieve the ultimate goals of the worldwide polio eradication program $[2,3,15]$.

The aim of this study was to detect the prevalence of poliomyelitis immunity in a representative population in a major urban center (São Paulo, Brazil), considered free of wild Poliovirus transmission since 1994 [9]. Poliomyelitis immunity (serotypes 1, 2 and 3 anti-polio antibodies) was highly prevalent $(>90 \%)$ in a representative population of São Paulo, Brazil, which is in accordance with the high vaccination coverage found for polio during a similar period in the country $(96.3 \%)$, in São Paulo state (114.7\%) and in the city (123.2\%) [26]. Also, only two individuals out of 1,037 $(0.2 \%)$ had no antibody response against any of the three polio types. None of the groups were below the threshold of $80 \%$ estimated prevalence of immunity to poliomyelitis. 
However, there were some significant variations in immunity prevalence among groups within the same strata. There were significant differences in the prevalence of anti-polio 1 immunity between sexes, which was confirmed by multivariate analysis, with a lower estimated immunity prevalence among men $(\mathrm{P}=0.003)$. The $\mathrm{OR}$ was $=2.439(95 \% \mathrm{CI}=1.370-4.348)$ for females in comparison with males. Although significant, the actual meaning of this information is uncertain and our model failed to explain this fact. We cannot refute a bias in the sampling methodology. However, the possibility of a real difference between males and females in this population should be further investigated.

Significant differences in the estimated prevalence of anti-polio 2 and 3 immunities were found among residence regions, according to the univariate model $(\mathrm{P}=0.029$ and 0.001 , respectively). The validity of the multivariate model applied to anti-polio 2 immunity was inadequate, probably due to the very low prevalence of non-immune individuals in this subgroup. However, this does not seem to be relevant from a public health standpoint, since the immunity prevalence estimate was extremely high. The multivariate model applied to antipolio 3 immunity indicated significant differences among residence regions $(\mathrm{P}=0.002)$. There were significant differences for the East, South and Downtown regions $(\mathrm{P}=0.006,0.018$ and 0.047 , respectively). Additionally, the East region compared to South and Downtown gave OR values of 3,000 (95\% CI = 1.627-5.533) and 3,544 (95\% CI $=1.484-8.459)$, respectively (Table 3c). Though it is not possible to draw any conclusions from the comparisons of immunity prevalence among the various regions, East region residents were more frequently immune to Poliovirus type 3 than South and Downtown residents (OR 3.000 and 3.544, respectively). The North region had the lowest anti-polio 2 immunity prevalence, according to the univariate model. Immunity level differences between regions could have epidemiological significance because of pockets of poverty within distinct city areas [27], however the exact meaning of this finding is still uncertain and is not explained by our study. These data are not conclusive, since the areas we evaluated do not necessarily characterize regions with low immunity prevalence. Also, detailed epidemiological evaluation of poverty regions and socioeconomic level are necessary to allow an objective comparison among distinct geographical areas. Nevertheless, pockets of low vaccination coverage either in geographical areas (poverty zones) or population groups (minorities) have been described within regions with high general coverage $[27,28]$. Since these findings might indicate areas with lower immunity prevalence in a resident population, further investigations should be made.

There were no significant differences among color/ race stratification groups.

In our opinion, age stratification deserves a more detailed analysis, since we found significantly higher prevalence of anti-polio 1 and 2 immunity among individuals $<18$ years old, compared to individuals $\geq 18$ years old ( $\mathrm{P}=0.002$ and 0.011 , respectively). Additionally, children under 6 years old had a $100 \%$ estimated immunity prevalence, which also is in accordance with the high vaccination coverage described [26]. Possibly this group made a strong contribution towards the general differences found for immunity prevalence among age groups. Also, another type of division of age groups $(<18,18-49$ and $\geq 50$ years) indicated a lower estimated anti-polio 1 immunity prevalence in the 18-49 year-old group $(0.919,95 \% \mathrm{CI}=0.896-0.942)$, compared to the $<18$ and $\geq 50$ groups $(\mathrm{P}<0.0001)$, possibly indicating a population with lower naturally acquired and vaccine immunities. Multivariate analysis $(P=0.003)$, confirmed a higher prevalence of anti-polio 1 immunity with $\mathrm{OR}=3.124(95 \% \mathrm{CI}=1.457-6.697)$ for the $<18$ year-old, compared with the $\geq 18$ year-old age group. Again, the validity of the multivariate analysis model was inadequate to evaluate any correlation for anti-polio 2 immunity, although as already stated, this does not seem to compromise the general results, since a very high estimated immunity level was observed for this subgroup. Even though support by multivariate analysis for age stratification was only observed for anti-polio 1 immunity, there seem to be sufficient significant findings evidencing differences among age groups. Although it is expected that a larger proportion of younger age groups would be exposed to vaccination campaigns, further investigations 
regarding age stratification and immunity appear to be necessary, especially among the $18-49$ year-old population.

Estimated immunity prevalence against all Poliovirus serotypes was quite high and the data seem to reinforce the idea that the National Vaccination Program was quite successful in the city of São Paulo, Brazil. Excellent levels of protective antibodies were detected in the sera of this population in all age groups, indicating that natural and/or vaccine immunities persist for a long period of time. The Brazilian National Vaccination Program is based solely on OPV use through routine and National Immunization Days (NIDs). These data seem to support the conclusion that this strategy has been sufficient to maintain high levels of serological immunity for a long period, since no age group was below the threshold of $84 \%$ estimated immunity prevalence (Graph 2). Nevertheless, this observation is more relevant to the part of the population that is exposed to vaccination campaigns (routine and NIDs), i.e. the younger age groups. Our study could not precisely establish the impact of naturally acquired immunity on this population, but it is reasonable to assume that among the individuals that were not exposed to vaccination efforts, natural immunity, along with passive transfer of OPV virus, have been the primary modes of immunity acquisition $[29,30]$.

However, there may be lower immunity prevalence within certain groups facing specific conditions and/or with specific viral serotypes [28, 31-33]. These findings could justify further surveys of the National Immunization Program results. A control study of vaccination results would enable the identification of specific population deficiencies and could point out needs for booster vaccines among determined groups [28].

The methodology used was characterized by a sample stratified by sex, age groups and residence region, representative of a large population $(9,626,894$ inhabitants of the city of São Paulo) [17]. This method was co-conducted by a private polling research and statistics institute and has previously been used in many public opinion polls with remarkable accuracy. This population-based sampling method was able to trace poliomyelitis immunity prevalence of an otherwise inaccessible group. Seroprevalence studies have been previously performed in other parts of the world and have proven their importance as a tool in polio eradication [28, 29, 34-36]. Estimation of poliomyelitis immunity prevalence made in a population-based survey could be used as an auxiliary tool for polio eradication programs. Areas close to achieving eradication, or where eradication has been recently achieved, might also benefit from this type of study as an orientation tool. However, further economic evaluations should be made to better establish the use of this method as a support for an eradication effort. Since we are closer to reaching polio eradication on a global scale [10], the development of auxiliary tools may help in evaluations of eradication results.

In conclusion, routine child immunization and NIDs with OPV have provided excellent levels of serologic immunity to poliomyelitis in the population of the city of São Paulo, Brazil, which has been exposed to an immunization program. As confirmed by stratification by age groups, antibodies persist for a long time irrespective of the mode of immunity acquisition. Despite the usefulness of insight into poliomyelitis immunity as an auxiliary tool for eradication, global eradication itself is the ultimate and only means of protecting any area against poliomyelitis.

\section{Aknowledgements}

The authors wish to thank Aventis-Pasteur/Brazil (former Pasteur-Merieux-Connaught) for the support given during study planning and development.

\section{References}

1. World Health Organization. World Health Assembly. Expanded Programme on Immunization. Geneva, Switzerland, 1988 (Resolution no. WHA41.28).

2. Birmingham M.E., Linkins R.W., Hull B.P., Hull HF. Poliomyelitis Surveillance: the compass for eradication. J Infect Dis, 1997;175(Suppl 1):S146-50.

3. Ward N.A., Milstien J.B., Hull H.F., Kim-Farley R.J. The WHO-EPI initiative for the global eradication of poliomyelitis. Biologicals 1993;21:327-33.

4. Foege W.L. Global polio eradication. Public Health Rev, 1993/94;21:3-10. 
5. Hull H.F., Ward N., Hull B.P., et al. Paralytic poliomyelitis: seasoned strategies, disappearing disease. Lancet 1994;343:1331-7.

6. Zhang J., Zhang L., Otten Jr M.W., et al. Surveillance for polio eradication in the People's Republic of China. J Infect Dis 1997; 175(Suppl 1):S122-34.

7. Center for Diseases Control and Prevention. Progress toward global eradication of poliomyelitis, 1997. MMWR 1998;47(20):414-9.

8. Pan American Health Organization. Director Announces campaign to eradicate poliomyelitis from the Americas by 1990. Bull Pan Am Health Organ, 1985;19:213-5.

9. Expanded Programme on Immunization. Certification of poliomyelitis eradication - the Americas, 1994. Wkly Epidemiol Rec 1994;69:293-5.

10. Center for Diseases Control and Prevention. Public health dispatch: Certification of poliomyelitis eradication Western Pacific Region October 2000. MMWR 2000; 50(01):1-3.

11. Center for Diseases Control and Prevention. Apparent Global Interruption of Wild Poliovirus Type 2 Transmission. MMWR 2001;50(12):222-4.

12. Andrus J.K., Banerjee K., Hull B.P., et al. Polio eradication in the World Health Organization south-east Asia region by the year 2000: midway assessment of progress and future challenges. J Infect Dis 1997;175(Suppl 1):S89-96.

13. Center for Diseases Control and Prevention. Progress toward global eradication of poliomyelitis - West Africa region, 1997 - September 1998. MMWR 1998;47(41): 883-6.

14. Center for Diseases Control and Prevention. Public health dispatch: Outbreak of poliomyelitis - Cape Verde, 2000. MMWR 2000;49(47):1070.

15. de Quadros C., Henderson D.A. Disease eradication and control in Americas. Biologicals 1993;21:335-43.

16. Center for Diseases Control and Prevention. Public Health Dispatch: Outbreak of poliomyelitis - Dominican Republic and Haiti, 2000. MMWR; 2000;49(48):1094-103.

17. São Paulo (State). Sistema Estadual de Análise de Dados. Anuário Estatístico do Estado de São Paulo. São Paulo, SEADE, 1995.

18. Brazil (Ministry of Planning and Development). Instituto Brasileiro de Geografia e Estatística. Anuário Estatístico. Brasília, IBGE, 1994.

19. São Paulo (State). Empresa de Planejamento da Grande São Paulo. Sumário de Dados da Grande São Paulo. São Paulo, EMPLASA, 1991.

20. Focaccia R, Conceição OJG, Sette Jr. H, et al. Estimated prevalence of viral hepatitis in the general population of the municipality of São Paulo, measured by serologic survey of a stratified, randomized and residence-based population. Braz J Infect Dis 1998;2(6):269-84.
21. Global Programme for Vaccines and Immunization. Vaccines Supply and Quality. World Health Organization. Manual of Laboratory Methods for Testing of Vaccines used in the WHO Expanded Programme on Immunization. Geneva, Switzerland, 1997;(Document no. WHO/VSQ, 97.04):65-71.

22. Altman DG. Practical statistics for medical research. $1^{\text {st }}$ Edition, London, Chapman and Hall, 1991:152-364.

23. Liu H.M., Zheng D.P., Zhang L.B., et al. Molecular evolution of a type 1 wild-vaccine poliovirus recombinant during widespread circulation in china. J Virol 2000;74(23):1153-61.

24. Naguib T., Yang S.J., Pallansch M., Kew O. Prolonged circulation of Sabin 2-derived polioviruses. In: Program and abstracts of progress in polio eradication: vaccination strategies for the end game. Geneva, Switzerland: International Association for Biologicals 2000.

25. Yoshida H., Horie H., Matsuura K., Miyamura T. Characterization of vaccine-derived poliovirus isolated from sewage and river water in Japan. Lancet, 2000;356(9240):1461-3.

26. Programa Nacional de Imunização (Fundação Nacional de Saúde, Ministry of Health, Brazil). Cobertura para poliomielite segundo unidades da federação e capitais. Brasília, DATASUS, 1998. Available at http://www.datasus.gov.br/cgi/pni/cpnimap.htm. Accessed January, 2001.

27. Expanded Programme on Immunization. Global Programme for Vaccines and Immunization. World Health Organization. Immunization policy. Geneva, Switzerland, 1996;(Document no.WHO/EPI/GEN 93.03):18-9.

28. Conyn-van Spaendonck M.A.E., de Melker H.E., Abbink F., et al. Immunity to poliomyelitis in the Netherlands. Am J Epidemiol 2001;153(3):207-14.

29. Chen R.T., Hausinger S., Dajani A.S., et al. Seroprevalence of antibody against poliovirus in inner-city preschool children. Implications for vaccination policy in the United States. JAMA 1996;275(21):1639-45.

30. Hull H.F., Lee J.W. Authors' reply: Oral poliomyelitis vaccines [Letter]. Lancet 1996;347(9013): 1495.

31. World Health Organization Collaborative Study Group on Oral Poliovirus Vaccine. Factors affecting the immunogenicity of oral poliovirus vaccine: a prospective evaluation in Brazil and the Gambia. J Infect Dis, 1995; 171(5):1097-106.

32. Gary Jr H.E., Freeman C., Peñaranda S., et al. Comparison of a monoclonal antibody-based IgM capture ELISA with a neutralization assay for assessing response to trivalent oral poliovirus vaccine. J Infect Dis 1997;175(Suppl 1): S264-7. 
33. Posey D.L., Linkins R.W., Couto Oliveira M.J., et al. The effect of diarrhea on oral poliovirus vaccine failure in Brazil. J Infect Dis 1997;175(Suppl 1):S258-63.

34. Diedrich S., Schreier E. Immunitatslage gegen Poliomyelitis. Polio-Serosurveillance 1993. Dtsch Med Wochenschr 1995;120(8):239-44.

35. Hanna J.N., Sexton W.L., Faoagali J.L., et al. Immunity to hepatits B, poliomyelitis and measles in fully vaccinated aboriginal and Torres Strait Island children. J Paediatr Child Health 1995;31(4):345-9.

36. Schoub B.D., MacAnerney J.M., van Middelkoop A., et al. A population based seroprevalence study in South Africa as a tool in the polio eradication initiative. Am J Trop Med Hyg; 1998;58(5):650-4. 